\title{
Lateral Tarsal Strip, Can It Be One Solution for All Types of Lower Eyelid Malposition? \\ Mohamed Al-Taher A. A ${ }^{1^{*}}$, Abd El Rahman Awadeen ${ }^{2}$ \\ ${ }^{1}$ Department of Ophthalmology, ${ }^{2}$ Department of Plastic, Burn, and Reconstructive Surgery, Faculty of Medicine (Boys), Al-Azhar University, Cairo, Egypt \\ *Corresponding author: Mohamed Al-Taher Abdel Wahab Abdel Atty, (+20)1010 444 972, drmaltaher75@yahoo.com
}

\begin{abstract}
Background: Due to the exact nature and complex anatomy of the eyelid, corrective surgeries require a comprehensive understanding of the anatomy as well as comprehensive preoperative planning and surgical enforcement.
\end{abstract}

Aim of the study: the present study was performed to evaluate the functional and cosmetic sequels of the lateral tarsal strip (LTS) in the correction of lower eyelid malposition

Patients and Methods: this prospective single-arm interventional study was carried on 27 patients (27 eyelids) with lower eyelid malposition, executed from January 2015 to December 2017. Patients with lower eyelid malposition in the form of entropion, ectropion, or laxity as a sequence of facial palsy, involutional changes, spastic entropion and lid laxity with failure of opposition to the artificial shell were included.

Results: Patients were 12 males and 15 females with a mean age of 59.37 \pm 10.27 years. Out of them, 17 (62.9\%), 8 (29.6\%), and 2 (7.4\%) were suffered from ectropion, entropion, and lower eyelid laxity, respectively. As for the functional and aesthetic outcomes, 10 (90.9\%) of patients with lower eyelid malposition, as a resultant effect of facial nerve palsy, have experienced complete healing of the epithelial defects; additionally, 4 (66.6\%) of involutional ectropion candidates showed improvement of the tearing eye. Eventually, 6 (22.2\%) participants developed postoperative complications.

Conclusion: LTS is a simple procedure which proved its safety and efficacy in the surgical correction of lower eyelid malposition of different types and etiologies

Keywords: Lateral tarsal strip, Eyelid Malposition, Entropion, Ectropion, Facial nerve palsy

\section{INTRODUCTION}

The lower eyelid tone and contour play an important role in maintaining corneal integrity and delivering tears along the lower eyelid margin. Eyelid skin is the thinnest existing in the body due to low dermis layer. Thus, it is the most vulnerable part of the skin to defects (1).

The resultant defects varied from deprivation of a small part of the superficial tissue up to the complete obscurity of the eyelid ${ }^{(2)}$. These defects may be evolved as a consequence of traumatic, iatrogenic or involutional changes ${ }^{(3)}$. It is worth noting that the deformity of the eyelids embraced special conditions such as entropion, ectropion and laxity of the lid which are crucial factors that change the direction and position of the eyelid ${ }^{(4)}$.

The most prevalent types of malposition are involutional ectropion and entropion owed by the fact that the involutional changes, due to aging, altered the fibrous and elastic tissues of the eyelid, principally those in the medial and lateral canthal tendons ${ }^{(4,5)}$, resulting in imbalance between eyelid tone and configuration on one hand, and the muscles of the eyelid on the other hand. The consequent secondary inflammatory changes, tarsal thickening, and punctum eversion aggravate the problem $^{(\mathbf{6})}$. In particular, epiphora, and corneal ulceration may manifest as a result of corneal exposure; additionally, the eventual cosmetic misconfiguration has a considerable psychological sequence in the patient's quality of life. Hence, early intervention is mandatory to avoid such complications ${ }^{(7)}$.

Owing to the precise nature of the eyelid and its complicated anatomy, eyelid correction surgeries necessitate an inclusive understanding of its anatomy along with comprehensive pre-operative planning and surgical enforcement ${ }^{(8)}$. Many surgical interventions have been demonstrated to remedy the eyelid malposition comprehending lateral tarsal strip (LTS), lateral Bick's shortening, everting sutures, and wedge excision $^{(9)}$. Even though these surgical procedures attained apparently prosperous outcomes, the preferable results were accomplished when the eyelid laxity was corrected ${ }^{(\mathbf{1 0})}$. The lateral tarsal strip approach is a promising oculoplastic approach on rectifying of the lower eyelid malposition. Not only did it preserve the lateral canthal angle and the sight as well, but it also avoids phimosis and disfigurement of the palpebral aperture ${ }^{(11)}$. 
The aim of the present study was to evaluate the effectiveness, functional and cosmetic sequels of LTS in the management of patients with lower eyelid malposition of different pathologies and variable etiologies as facial nerve palsy, ectropion, entropion of involutional and spastic nature and lid laxity.

\section{PATIENTS AND METHODS}

This prospective single-arm interventional study included a total of 27 patients (27 eyelids) with lower eyelid malposition, attending at the Department of Ophthalmology in co-operation with the Department of Plastic Surgery at Bab Elshaarya, and Damietta, AlAzhar University Hospitals, Egypt. This study was conducted between January 2015 to December 2017.

Written informed consent were obtained from all the patients in accordance with the Declaration of Helsinki and after complete explanation of the possible adverse events and risks of the technique.

\section{Inclusion criteria:}

Patients with lower eyelid malposition in the form of entropion, ectropion, or laxity as a sequence of facial palsy, involutional changes, spastic entropion and lid laxity with failure of the opposition to the artificial shell were included.

\section{Exclusion criteria:}

participants with congenital, cicatricial, recurrent lower eyelid malposition, patients with an intense lack of retractors functions or who were grade 4 medial canthal tendon laxity were omitted. Similarly, patients who were submitted to previous lower eyelid surgery and participants who suffered from excessive horizontal laxity of the lower eyelid as a sequence of the floppy eyelid syndrome were also excluded.

\section{Pre-operative evaluation}

All candidates were subjected to pre-operative evaluation which comprised pinch test to measure the horizontal eyelid laxity. To illustrate, the lower eyelid was promptly towed away from the globe, whereby the distance between the lower eyelid and eye was appreciated. Lower eyelid laxity was established, when the distance between the cornea and lower eyelid exceeded $6 \mathrm{~mm}$. Herein, we categorized its degree into a slight (7-10mm), moderate (11-14mm), and severe $(\geq 15 \mathrm{~mm})$ laxity. Furthermore, the function of the orbicularis and retractors muscles of the lower eyelid was assessed, whereas orbicularis muscle hypertrophy was diagnosed by clinical examination of the muscle at both contraction and resting states. In addition, we evaluated the retractors function by measuring the distance between lower eyelid crease and the lash line. Hence, we deemed normal muscle function when the lower eyelid skin crease $<4 \mathrm{~mm}$, whilst patients with lid crease at $4-6 \mathrm{~mm}$ and $7-8 \mathrm{~mm}$ were assorted as slight and moderate dysfunction, respectively. Complete preoperative ophthalmic examination was executed including slit lamp biomicroscopy with special concern to corneal epithelial integrity and visual acuity.

\section{Surgical procedure:}

\section{All patients were operated under local anesthesia:}

We injected approximately 3-5 $\mathrm{ml}$ of a blend of $2 \%$ Lidocaine along with epinephrine in a ratio of 1:200.000 using a 27-gauge needle in the lateral canthal angle, lateral eyelid and over the lateral orbital rim. To enhance the homeostasis process, a lateral canthotomy was implemented after crushing of the outer canthus with a mosquito forceps, the Westcott scissors was used in a vertical direction to get cutting which extended for nearly $10 \mathrm{~mm}$. Furthermore, we extracted the lateral canthal tendon attachments from the lateral orbital rim via oblique and nasally directed scissors to cut through the lower crus of the lateral canthal tendon in order to mobilize the lower eyelid and to identify the orbital periosteum. Consequently, we executed approximately $6-10 \mathrm{~mm}$ full-thickness horizontal cut in the lower eyelid. Another higher subciliary incision line of the same length of the previous horizontal cut was done, excision of the skin, subcutaneous tissue and the underlying orbicularis to get the tarsal strip bared and fashioned, with the help of bipolar diathermy the lid margin corresponding to the LTS was de-epithelialized and lashes were ablated as well as the conjunctiva corresponding to the LTS.

We pulled the strip overhead the angle of the lateral canthus under mild traction to depict extra length that was to be removed. Besides, we sutured the strip to the periosteum which presented on the interior aspect of the lateral wall of the orbit using a Polyester 4/0 double armed non-absorbable suture throughout a half-circle needle. Additionally, we passed the two needles out of the periosteum either just into the rim of the orbit or via the stump of the lateral canthal tendon. We sealed the sutures to overcome the possibility of post-operative dehiscence, which induced recurrence of the initial condition. Finally we closed the orbicularis muscle and the skin in two separate layers using 6-0 vicryl. (Figure.1) 


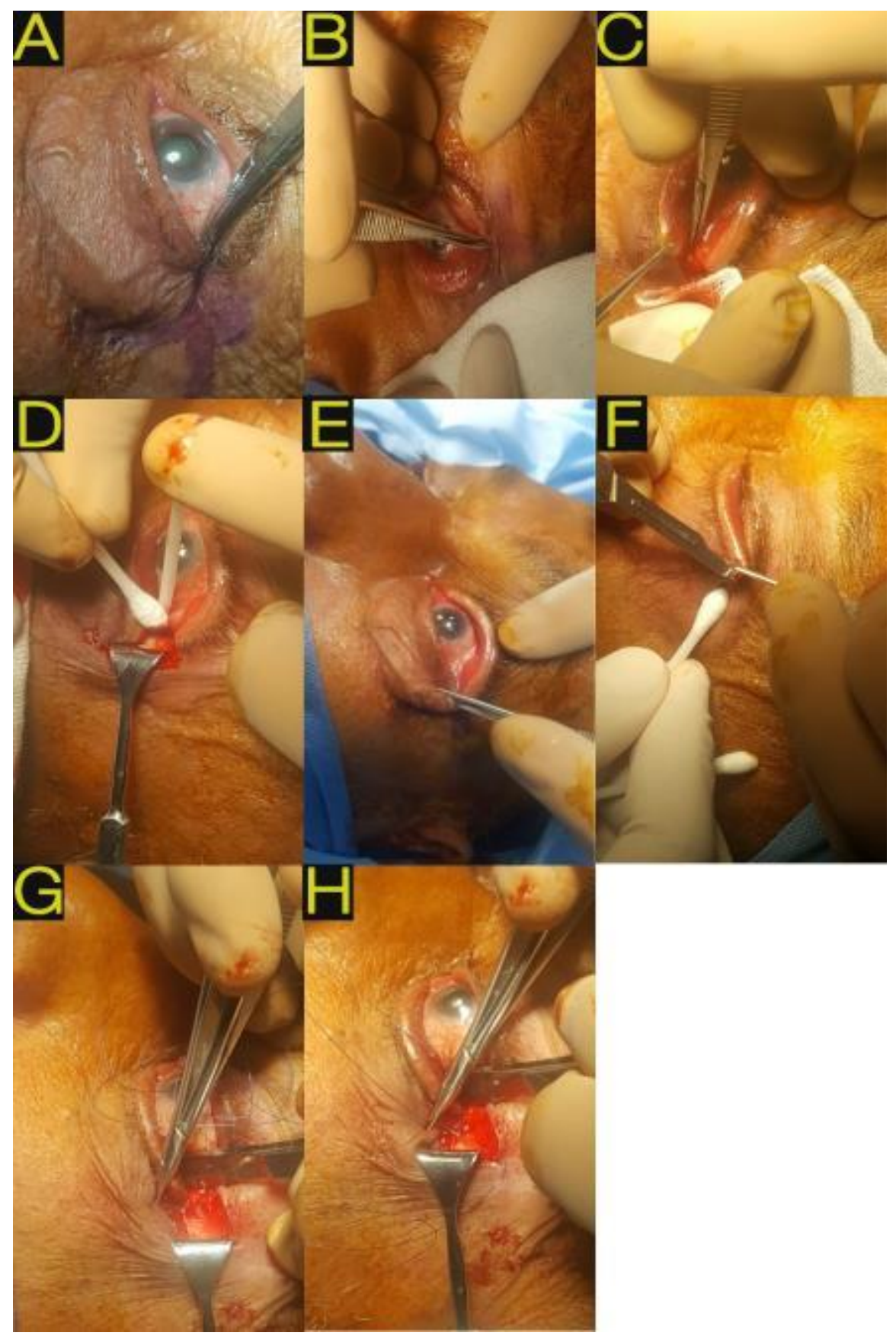

Figure (1): The surgical procedure of lateral tarsal strip (LTS)

A. Crushing of lateral canthus, canthotomy preparation. B. Lateral canthotomy. C. Lateral cantholysis and division of lower crus of lateral canthal tendon. D. Exposure of lateral orbital rim periosteum. E. Assess amount of LTS to be excised F. Fashioning of LTS. G. First pass of mattress suture in the periosteum. H. Second pass of mattress suture in the periosteum 


\section{Post-operative management:}

All participants were prescribed lubricants eyedrops, combined tobramycin+dexamethasone eye drops and ointment, systemic antibiotics, systemic nonsteroidal anti-inflammatory drugs and alpha chymotrypsin tablets for 21 days after the surgery; subsequently, the sutures were removed 10 days afterward. The followup protocol was established to reveal early and late functional and aesthetic outcomes; thereafter, clinical estimation was performed at 1,3, and 7 days, 2 and 4 weeks, and at 1,3,6, and 12 months after the surgery. For each visit, to reveal any adverse events, patients were submitted to rigorous history taking including symptoms of redness, pain, tearing, photophobia, visual deterioration, and foreign body sensation; moreover, a complete ophthalmic evaluation was done to discover any signs of corneal deterioration (aggravation of the previous abrasions or ulcers, corneal haze or opacification), chronic conjunctivitis or punctate erosions of the epithelium. Patients who developed suture granuloma were submitted to subsequent excision one month later, whilst participants who suffered from stitch abscess were treated with broad-spectrum antibiotics. Eventually, candidates who experienced suture lysis with recurrence were subordinated to revision surgery within one month after the initial intervention.

1.

\section{Statistical analysis}

Statistical analysis was performed using MedCalc software version 14.8 (MedCalc Software, Mariakerke, Belgium). Normally distributed data was elucidated in the term of mean and standard deviation (SD), while categorical data was symbolized as number and percentage.

\section{RESULTS}

An overall 27 participants (27 eyelids) with lower eyelid malposition, who fulfilled the inclusion criteria were eligible for enrolment in the current study. There were 12 males and 15 females with a mean age of $59.37 \pm 10.27$ years. Out of them, 17 $(62.9 \%), 8(29.6 \%)$, and $2(7.4 \%)$ patients were suffered from ectropion, entropion, and lower eyelid laxity, respectively. Furthermore, lower lid malposition was a sequel of facial nerve palsy in $11(40.7 \%)$ participants, whilst involutional changes contributed to 12 (44.4\%) mispositioned eyelids; moreover, spastic entropion and lower lid laxity were the underlying etiologies of 4 (14.8\%) lower eyelids malposition(Table.1 and Figure 2).

Table (1): Demographic characteristics of the included participants.

\begin{tabular}{|c|c|}
\hline Total eyelids (patients) & 27 \\
\hline Age & $18-80$ years \\
\hline Sex & 12/27 Males, 15/27 Females \\
\hline Types of malposition & $\begin{array}{l}\text { Ectropion: } 17 / 27 \\
\text { Entropion: } 8 / 27 \\
\text { Lower Eyelid laxity: 2/27 }\end{array}$ \\
\hline Etiology of malposition & $\begin{array}{l}\text { Facial nerve palsy } 11 / 27 \\
\text { Involutional ectropion } 6 / 27 \\
\text { Involutional entropion } 6 / 27 \\
\text { Spastic entropion } 2 / 27 \\
\text { Lower lid laxity with failure of apposition to the artificial shell, Rounding of } \\
\text { the lateral canthus } 2 / 27\end{array}$ \\
\hline
\end{tabular}




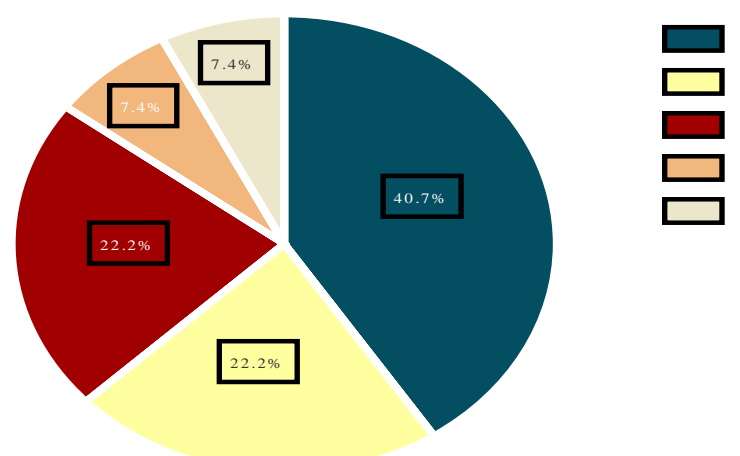

Facial nerve palsy

Involutional ectropion

Involutional entropion

Spastic entropion

Low er lid laxity

Figure (2):Pie chart demonstrated the underlying etiologies of lower eyelid malposition among the included patients.

As for the functional and aesthetic outcomes, 10 $(90.9 \%)$ of patients with lower eyelid malposition, as a resultant effect of facial nerve palsy, have experienced complete healing of the epithelial defects; additionally, $4(66.6 \%)$ of involutional ectropion candidates showed improvement of the tearing eye. Consequently, all patients with involutional entropion not only did they experience improvement of the tearing eye, but they also showed amelioration of the irritation symptom. Similarly, all patients with spastic entropion encountered considerable improvement in the tearing eye and discomfort symptoms.
For patients with lower lid laxity, our results revealed that all of them regainedthe optimal tone of the lower eyelid, well apposition and holding the artificial shell as well as correction of the lateral canthus contour(Figures.3, 4, 5 and 6). On the contrary, out of 27 patients, $6(22.2 \%)$ participants developed postoperative complications counterpart suture granuloma, $2(7.4 \%)$, stitch abscess, 2 (7.4\%), and suture lysis with recurrence of laxity and ectropion in $2(7.4 \%)$ cases (Figure 7).

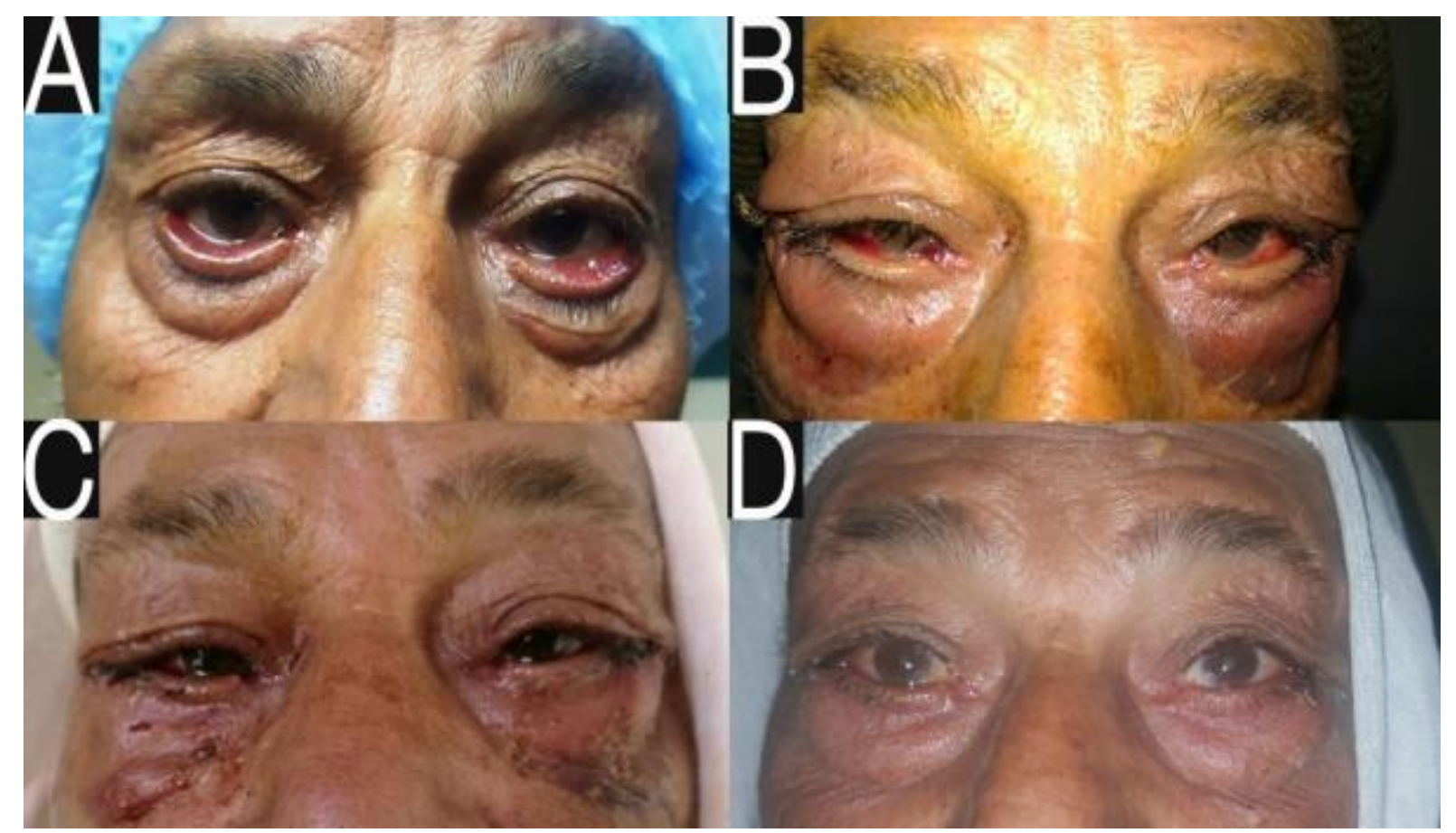

Figure (3): A case of bilateral involutional ectropion A. Just pre-operative. B. Two-days post-operative. C. Twoweeks post-operative. D. Three-months post-operative 


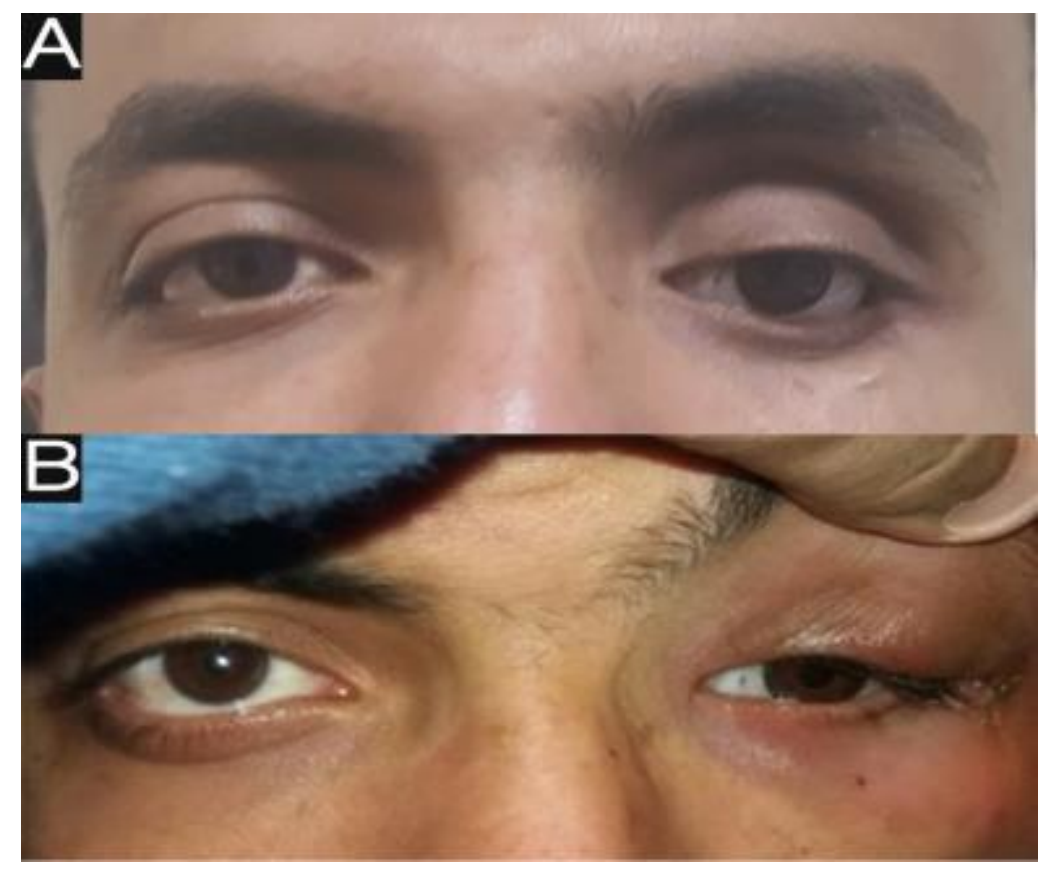

Figure (4): Male patient represented by unsupported shell by the lower eyelid of the left eye A. Pre-operative unsupported shell. B. Post-operative well supported shell

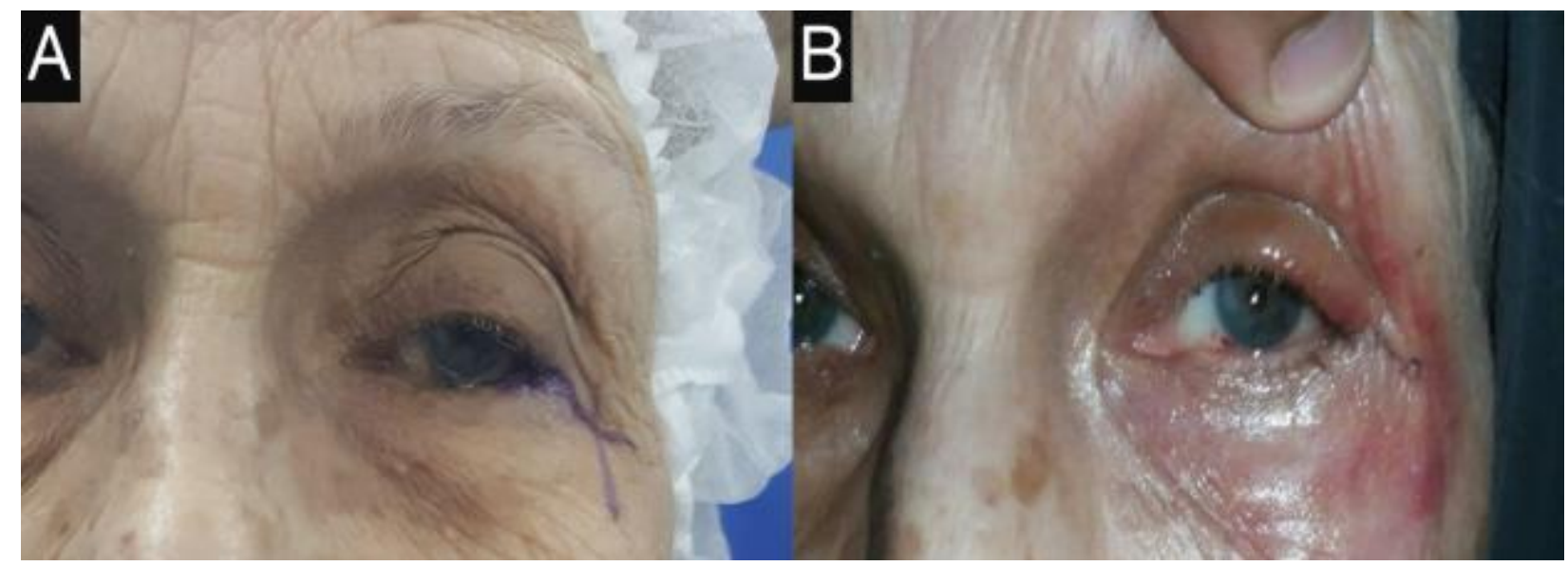

Figure (5): Female patient presented with left spastic entropion.

A. Pre-operative B. Post-operative

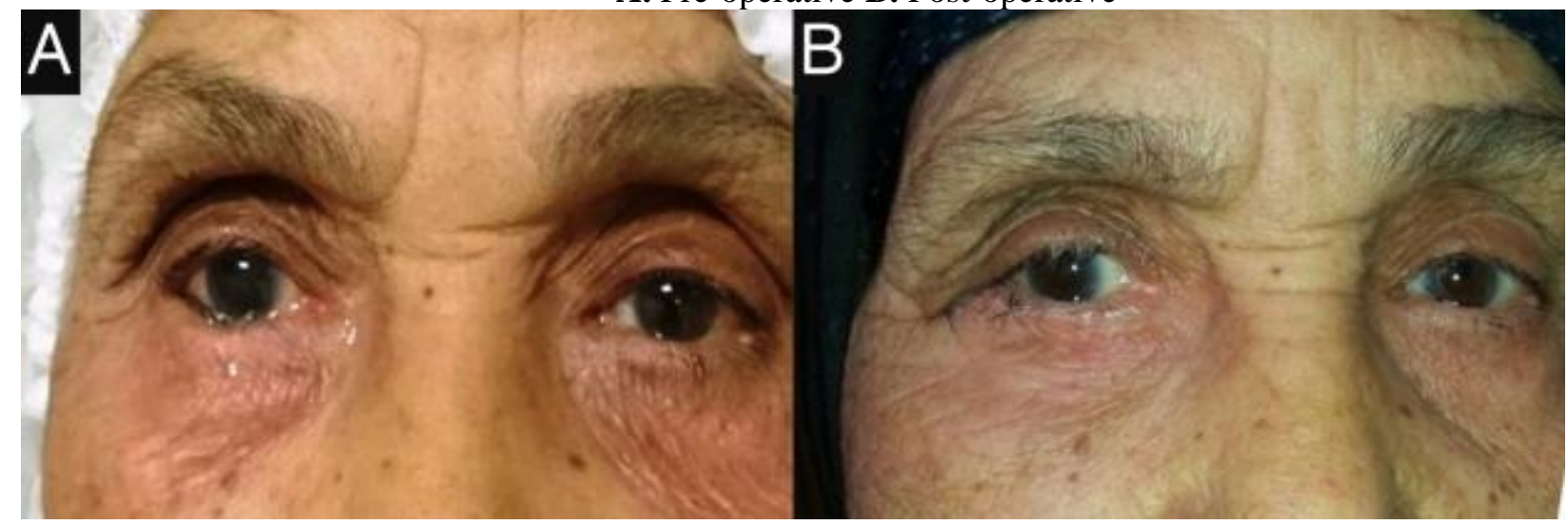

Figure (6): Female patient presented with right involutional entropion.

A. Pre-operative B. Post-operative. 


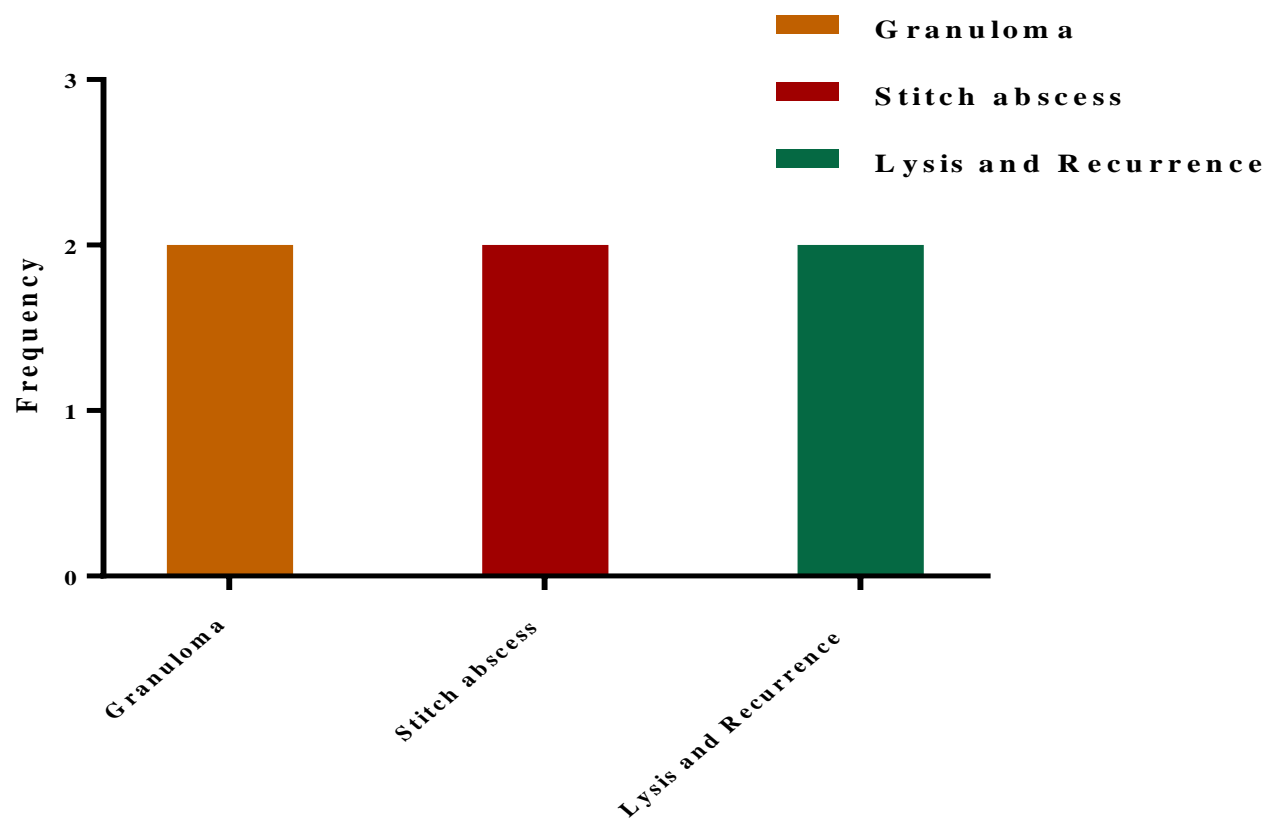

Figure (7): Bar chart delineated the pattern of post-operative complications.

\section{DISCUSSION}

Throughout literature search, abundant surgical interventions have been depicted for correction of the lower eyelid malposition such as lateral tarsal strip, lateral and medial canthal tendon plication, lateral canthal tendon plication or medial canthal resection $^{(\mathbf{1 2})}$. To date, LTS procedures were described initially by Anderson et al. $^{\text {(13) }}$ after modification of the Tenzel procedure. After that, this technique became probably the most common intervention for eyelid malposition correction ${ }^{(4)}$. Of note, the LTS procedure accomplished notability,more than the other procedures, in the management of lower eyelid malposition,specially in the merits ofprompt rehabilitation and better cosmetic outcomes ${ }^{(\mathbf{1 4})}$.

The current study is an unrivaled investigation, which evaluated whether LTS solely is felicitous approach in the management of patients with lower eyelid entropion, ectropion, and laxity regardless of the underlying etiology. Interestingly, our resultsarticulated that LTS approach accomplished the desired functional and aesthetic outcomes among patients with distinct genre of lower eyelid malposition, principally those with involutional or spastic entropion. This is because the LTSadvantages embraced restoration of the normal anatomy and integrity of the lid and protection against lid notching, horizontal phimosis, and recurrence of the laxity which reflected dramatically on the patient's outcomes $\left.{ }^{(15,} \mathbf{1 6}\right)$. Consequently, wetighten the eyelid effectively which reverberated significantly in the orbicularis muscle function to boost the tears to the punctum; additionally, this procedure attained the optimal horizontal tightening and managed the dehiscence of the retractors without alteration of the lamellar tissue ${ }^{(\mathbf{1 7})}$. Furthermore, two patients in our study have experienced recurrence and were subordinated to revision surgery with LTSat 2 and 3 weeks respectively, after the primary surgery, both cases achieved adequate correction but with modified postoperative medications (prescription of broad-spectrum antibiotics for 10 days with no steroid eye drops or ointments). Thereafter, we can postulate that LTS approach is also appropriate approach in the remedy of the recurrent cases; however, further studies are required to appreciate this conclusion.On the contrary, in such patients, some studies supposed to use an extra approach such as transconjunctival retractor placation to achieve dramatic effectiveness, particularly among patients with lower eyelid medial ectropion ${ }^{(\mathbf{1 7})}$

Regarding post-operative complications, our study revealed that a small number of patients had experienced post-operative adverseevents; however, this number was noticeably well beneath Kam $\boldsymbol{e t} \boldsymbol{a l} .{ }^{(\mathbf{1 8})}$ study who reported $47 \%$ post-operative complications rate. Furthermore, Rocca $\boldsymbol{e t} \boldsymbol{a l}^{\left({ }^{(19)}\right.}$ notified that inadequate positioning of the strip leads to a gap 
between the upper and lower eyelids which lead to ocular exposure. However, in our study, neither of these complications was reported.

The relatively small sample size, the lack of randomization, obscurity of comparison group, and the substantial heterogeneity between the included participants regarding the underlying patho-etiologies and types of lower eyelid malposition may hinder the evidence of the study.

\section{CONCLUSION}

It could be concluded that the LTS is a standalone procedure, which accomplished a competent functional and cosmetic consequences in patients with lower eyelid malposition. Nevertheless, high quality randomized clinical trials are mandatory to address the limitations of our study.

\section{REFERENCES}

1. Niamtu J (2016): Cosmetic Facial Surgery-E-Book: Elsevier Health Sciences. https://www.elsevier.com/ books/cosmetic-facial-surgery/niamtu/978-0-32307400-1

2. Guthrie AJ, Kadakia P and Rosenberg J (2019): Eyelid Malposition Repair: A Review of the Literature and Current Techniques. Seminars in plast Surg., 33(02): 092-102.

3. Nagaraju G, Chhabria KP and Samhitha H(2015):Dynamics of Lower Lid Malpositions. J of Evid based Med and Healthc., 2(9)1295-301.

4. Hahn S, Desai SC (2016): Lower lid malposition: causes and correction. Facial Plast SurgClin.,24(2)16371.

5. Kam K, Cole C, Bunce Cet al. (2012): The lateral tarsal strip in ectropion surgery: is it effective when performed in isolation? Eye, 26(6)827.

6. López-García JS, García-Lozano I, Giménez-Vallejo C et al. (2017): Modified lateral tarsal strip for involutional entropion and ectropion surgery. Graefe's Archfor Clin and Exp Ophthalmol.,255(3)619-25.
7. Chhabria KP (2015): Eyelid malposition: lower lid entropion and ectropion. Medicina (Kaunas), 42(11):8814.

8. Alghoul M, Pacella SJ, McClellan WT, Codner MA (2013): Eyelid reconstruction. Plast and Reconstructive Surg.,132(2):288-302.

9. Codner MA, McCord CD (2016): Eyelid and periorbital surgery: Kerala J Ophthalmol., 29:160-7.

10. Vahdani K, Ford R, Garrott H, Thaller VT(2018): Lateral tarsal strip versus Bick's procedure in correction of eyelid malposition. Eye,32(6):1117-1122.

11. Dulz S, Green S, Mehlan J, Schüttauf F, Keserü M (2019): A comparison of the lateral tarsal strip with everting sutures and the Quickert procedure for involutional entropion. Acta Ophthalmol. doi: 10.1111/aos.14093.

12. Collin JR (2006): A manual of systematic eyelid surgery: Elsevier Health Sciences. https://www.elsevier.com/books/a-manual-ofsystematic-eyelid-surgery/collin/978-0-7506-4550-8

13. Anderson RL, Gordy DD(1979):The tarsal strip procedure. Arch of Ophthalmol.,97(11): 2192-6.

14. Barrett RV, Meyer DR (2012): The modified Bick quick strip procedure for surgical treatment of eyelid malposition. Ophthal Plast and Reconstructive Surg.,28(4):294-9.

15. Kim KH, Baek JS, Lee Set al. (2017): Causes and Surgical Outcomes of Lower Eyelid Retraction. Korean J of Ophthalmol.,31(4): 290-8.

16. Olver JM(1998): Surgical tips on the lateral tarsal strip. Eye,12 ( Pt 6):1007-1012.

17. Fong KCS, Mavrikakis I, Sagili Set al.(2006): Correction of involutional lower eyelid medial ectropion with transconjunctival approach retractor plication and lateral tarsal strip. Acta Ophthalmol Scandinavica, 84(2):246-249.

18. Kam KY, Cole CJ, Bunce Cet al.(2012): The lateral tarsal strip in ectropion surgery: is it effective when performed in isolation?. Eye, 26(6):827-832.

19. Della Rocca DA (2007): The lateral tarsal strip: illustrated pearls. Facial plast Surg.,23(3):200-202. 